\title{
Interferometry with Large Molecules: Exploration of Coherence, Decoherence and Novel Beam Methods
}

\author{
Markus Arndt, Lucia Hackermüller, and Elisabeth Reiger \\ Institut für Experimentalphysik, Universität Wien, Boltzmanngasse 5 \\ Received on 25 January, 2005
}

\begin{abstract}
Quantum experiments with complex objects are of fundamental interest as they allow to quantitatively trace the quantum-to-classical transition under the influence of various interactions between the quantum object and its environment. We briefly review the present status of matter wave interferometry and decoherence studies with large molecules and focus in particular on the challenges for novel beam methods for molecular quantum optics with clusters, macromolecules or nanocrystals.
\end{abstract}

\section{INTRODUCTION}

Recent years have seen tremendous progress in experiments demonstrating the very foundations of quantum physics with systems of rather large size and complexity. The present article focuses on matter wave interferometry, which clearly visualizes the essence of the quantum superposition principle for position states of massive particles. Related experiments have been pursued for more than seventy years by now. First demonstrated in the diffraction of electrons [1] as well as with atoms and dimer molecules [2] the delocalized behavior of matter has recently also been studied in mesoscopic systems. The coherent dynamics of large ultra-cold ensembles of Bose condensed atoms [3] as well as the interferometry of electrons - be it using magnetic edge states [4] or superconducting circuits [5] in cold solids - are famous examples for that. Our own work expands the range of related experiments into the realm of hot covalently bound molecules, currently with masses up to $1600 \mathrm{u}$, and internal temperatures as high as a more than thousand Kelvin.

First studies on the diffraction of large molecules followed the very idea of Young's double slit experiment [6], and were successfully performed both with hot $\mathrm{C}_{60}$ fullerene molecules [7] and with cold He clusters [8]. In order to extend these investigations for objects of even higher mass and complexity many new techniques had to and still have to be developed. Any coherence experiment requires a suitable source, an optimized interferometer design and an efficient detector. It is also of particular importance to identify and eliminate possible causes of decoherence, which will limit the observability of genuine quantum phenomena.

In the present paper we summarize the status of coherence and decoherence experiments with big molecules in Section II. Section III is then dedicated to ideas and experiments exploring both laser desorption and electrospray methods for generating beams from small bio-dyes, such as porphyrins, up to proteins and nanocrystals. We discuss the present challenges and future prospects of such sources for quantum experiments with nanosized material.



FIG. 1: Talbot Lau interferometry with molecules. a) $\mathrm{G}_{1}$ prepares the required spatial coherence for diffraction at $\mathrm{G}_{2}$. The emerging interference pattern is scanned by the spatially resolving mask $G_{3}$. b) Collisions with residual gas molecules result in an entanglement between the colliding partners but also in a recoil of the diffracting molecule. c) Thermal emission of light is an intriguing aspect of molecular decoherence. As in the collision example, interference becomes unobservable if the shared information suffices (in principle) to locate the molecule inside the interferometer.

\section{STATUS OF COHERENCE AND DECOHERENCE EXPERIMENTS WITH MOLECULES}

In contrast to double-slit far-field diffraction, which is the textbook example for the demonstration of the wave nature of light or matter, our recent experiments have always employed near-field Talbot-Lau interferometry [9-11]. Its main advantages are two-fold: On the one hand it offers a more 
than thousand-fold higher material throughput and count rate. This is due to the fact that this particular setup can accept uncollimated, i.e. spatially incoherent, input beams. On the other hand near-field effects have a good mass-scaling behavior: at a given interferometer length, the required diffraction grating constant decreases only with $\sqrt{\lambda_{d B}}$ instead of $\lambda_{d B}$, as was already emphasized by Clauser [12]. For our experiments with Fullerenes, to be reported below, this means for instance that we could work with a grating period of $1000 \mathrm{~nm}$ in the near-field while we had to use a $100 \mathrm{~nm}$ structure for far-field diffraction of the same molecular beam.

The idea of our setup is shown in Fig. 1a). Uncollimated, but velocity selected $(\Delta v / v \sim 10-15 \%)$ molecules pass a first grating which imprints a spatial periodicity of $990 \mathrm{~nm}$ onto the transverse beam profile. The grating openings of $450 \mathrm{~nm}$ impose a spatial coherence on the beam, which suffices to coherently illuminate a few neighboring slits on the second grating. Diffraction at this second structure leads to a periodic molecular density pattern at the position of the third grating. The period of the emerging interference pattern, turns out to be equal to the grating constant if the distance $L$ between subsequent gratings corresponds to the Talbot length $L_{T}=g^{2} / \lambda[14,15]$. In order to prove the existence of the interference fringes and to quantitatively assess the visibility we scan the third grating transversely across the molecular beam. Whenever the density pattern and the mask have synchronized positions a maximal number of molecules can pass the grating and move on to the detector. Else they are partially blocked by the grating and the count rate is decreased. The resulting interference pattern is shown in Fig. 2. A complete proof of the wave nature of matter also has to verify that the observed molecular density pattern depends on the wavelength, i.e. on the velocity of the molecules. And this has indeed be shown quantitatively in our experiments [16].

Using this setup we could successfully demonstrate the



FIG. 2: Interference fringes of tetraphenylprophyrin after passage through the Talbot Lau interferometer. The contrast of about $30 \%$ in this particular picture is as high as expected for the given interferometer geometry and molecular velocity distribution[13]. wave-nature of various large molecules [13], among them the fullerenes $\mathrm{C}_{70}$, the biodye tetraphenylporphyrin (TPP) as well as the fluorinated fullerene $\mathrm{C}_{60} \mathrm{~F}_{48}$, shown in Fig. 3. The experiments clearly prove that the quantum superposition principle still holds for rather complex objects.

But we can also investigate in a quantitative way under which circumstances molecules will appear as classical balls. The corresponding work has already been published in great detail [13, 17-19]. We will therefore only briefly summarize the general idea of these experiments. Our experiment is a pure 'de Broglie interferometer', i.e. the internal state of the particle is the same in all possible arms. But which-path information can be obtained by emission or scattering of particles. It turns out that collisions with molecules in the background gas of the vacuum chamber is a most naturally occurring decoherence scheme in matter wave interferometry. The colliding particle changes its path in dependence on the exact location of the binary encounter and thus carries information about the collision point (see Fig. 1b). But even in the absence of any residual gas decoherence can occur if the interfering object is sufficiently complex and hot. Such a molecule may emit thermal light, ranging from the visible to the infrared domain. A single photon with a wavelength comparable to the separation of two superposed wave-packets of the diffracting molecule suffices to transmit precise information about the location of the emitting particle and thus to destroy the interference pattern. With increasing temperature the probability of photon emission increases and the average wavelength decreases. Both effects smoothly reduce the molecular interference contrast.

From quantitative experiments with a number of different background gases as well as from our thermal investigations with Fullerenes we conclude that the decoherence limits of matter interferometry are still several orders of magnitude away from realistic technical limitations for earth-bound experiments. It is therefore highly desirable to develop new sources which are suitable for coherence studies with much larger species than those used so far.



FIG. 3: Gallery of molecules that showed their wave-nature in Talbot-Lau interferometry. Left: Fullerene, $\mathrm{C}_{70}$. Middle: Tetraphenylporphyrin, a variant of a typical biodye. Right:Fluorofullerene $\mathrm{C}_{60} \mathrm{~F}_{48}$. 


\section{TOWARDS NEW SOURCES FOR QUANTUM EXPERIMENTS WITH LARGE MOLECULES}

\section{A. General considerations}

In the previous paragraph we have avoided technical details such as how to make fullerenes, how to bring them into the gas phase, how to improve the coherence of the beam or how to detect the molecules. In the present section we want to focus on one particularly challenging point and discuss the source requirements for coherence experiments with objects containing several hundred to hundred thousand atoms.

It is clear that the requirements on the source quality are much affected by our detection capabilities. An interferogram, composed of 10,000 particles detected in the right place and in a narrow velocity interval could already yield a sufficiently good signal-to-noise ratio to create an interference pattern.

But, for instance the electron impact ionization schemes employed in the latest molecule interference experiments [13] had a total detection efficiency of only $0.00001 \ldots 0.001$ and even our laser ionization method did not register more than a few percent of all fullerenes [20]. More promising recording schemes, based on optical and surface probe methods, are currently being investigated.

Also the particular interferometer that we choose will strongly affect the source requirements. It has already been mentioned that near-field interferometry is superior to far-field diffraction as it does not require a collimated beam. The count rate in the near-field setup [16] exceeded that of the far-field [7] by a bit more than a factor one thousand in our successful experiments with $\mathrm{C}_{70}$.

Even for a fixed interferometer type, subtle technical details - such as regularity of the available nanomechanically fabricated gratings - will determine the useful grating area and thus the totally transmitted number of molecules.

These arguments already show that any specific experimental prediction has to consider all details with great care. In the following we can therefore only describe qualitatively the prospects and challenges, which have to be sorted out in our ongoing exploration of new sources for matter interferometry.

Volatilizing massive objects is generally an outstanding task, as the high mass, the large polarizability or even permanent dipole moments of many nanosized particles usually let these objects stick to their neighbors. And even if the particles can be brought into the gas phase, there are still several conditions to be fulfilled to make them useful for coherence experiments.

a. The beam flux should be high and sufficiently stable in order to get a good signal-to-noise ratio and to minimize the influence of drifts and vibrations in interferometers with nanosized diffraction structures. But for the sake of the argument of single-particle interference the beam density has also to remain sufficiently small. In our recent fullerene experiments the average beam density was as low as $1000 \mathrm{~cm}^{-3}$. The corresponding average separation between two molecules of $1 \mathrm{~mm}$ exceeds the range of typical van der Waals forces by about a factor one thousand. b. The beam velocity should be small in order to achieve long de Broglie wavelengths and large diffraction angles. However slowing of molecules is still an outstanding experimental challenge and longitudinal slowing alone will not improve the source quality as it degrades the beam collimation.

c. The beam divergence in far-field grating diffraction must be smaller than the diffraction angle. This is summarized by the condition $\Theta_{\text {coll }}<h / g m v$, with $\Theta_{\text {coll }}$ the collimation angle, $m$ and $v$ the mass and the velocity of the diffracted particle and $g$ the period of the diffraction grating. For fullerenes at $200 \mathrm{~m} / \mathrm{s}$ the required collimation was as small as $20 \mu \mathrm{rad}$. This criterion can also be written as $v_{T}<h / g m$ indicating that the transverse velocity must be very small. In far-field diffraction of the fullerenes $v_{T}$ was as small as $2 \mathrm{~mm} / \mathrm{s}$ given by the tight collimation. If we were to achieve such a small transverse velocity by genuine cooling this would correspond to a temperature below $T<h^{2} / g^{2} m k_{B}$, i.e. several microkelvin (!) for the case mentioned above. This has to be contrasted with the present source temperature of about $1000 \mathrm{~K}$, which is required for sublimating Fullerenes to sufficient vapor pressures. In near-field interferometry the collimation was often limited to about $1 \mathrm{mrad}[13,16]$. This was mainly determined by the available detector size. But also other technical restrictions, such as the largest useful size of available mechanical nanostructures currently limit the divergence angle to a few mrad.

d. The longitudinal velocity spread has to be sufficiently small to avoid chromatic phase averaging of the interferogram. Minimizing $\Delta v$ is also particularly important in the presence of external force fields, such as the gravitational field of the earth or the van der Waals potential between the interfering molecule and the wall of the diffraction grating. The corresponding beam deflection in itself can be calibrated and even be used for measuring the deflecting potentials. However, a significant velocity spread will cause an incoherent average of shifted interference patterns, which reduces the interference contrast. Both the longitudinal and transverse velocity spread can easily be limited using various selection methods. But the available phase space density in the beam will set a limit, as in the end a finite count rate must also be maintained.

e. Monodispersity, i.e. the presence of a single type of particles in the beam, is a desirable but not an indispensable source criterion. Postselection and identification can also be realized in the detector using either mass or fluorescence spectroscopy.

f. Vacuum compatibility is important for near-field interferometers with molecules beyond the $1000 \mathrm{u}$ range. Pressures significantly better than $10^{-7}$ mbar are required to avoid collisional decoherence (see $[17,21]$ ). For proteins this value has to be decreased by up to two orders of magnitude. The source must therefore usually be separated from the interferometer through one or even several differential pumping stages.

g. Neutral molecules appear to be least affected by electro-magnetic dephasing and decoherence. It is therefore most natural to insist on neutrality. On the other hand, we will see below that it is much easier to fulfill some of the other requirements if we accept charged particles - at least in some 




FIG. 4: Mass Spectrum of thermally laser desorbed neutral porphyrin which was subsequently ionized using a second pulsed uv laser. The graph shows that a rather monodisperse, intense signal can be generated and detected.

stage of the experiment.

Both the physics of molecular beams [22] as well as experiments with ultra-cold atomic beams [23] are well-established by now. And all our macromolecule interference experiments up to date were performed with a conventional effusive source in which a solid material was sublimated at elevated temperatures.

However this simple technique seems not be applicable to particles beyond $10.000 \mathrm{u}$ and none of the presently known beam manipulation methods fulfills already all of the above mentioned criteria for particles in this high-mass regime. In particular the application of various laser cooling schemes, which turned out to be the key to the success of cold atom experiments, is largely hampered by the complex internal structure of large molecules.

In the following we will briefly discuss two methods whose potential for quantum experiments is currently being explored: electrospray ionization and laser desorption. They are adopted from mass spectroscopy where they are widely used for the volatilization of macromolecular ion beams [24, 25].

\section{B. Laser desorption}

\section{Thermal laser desorption, $T L D$}

Particles with both a high thermal stability and a high optical absorption cross section can be evaporated from bulk material using a short laser pulse.

Figure 4 shows a mass spectrum of tetraphenylporphyrin (TPP) which was volatilized by a $N_{2}$-laser pulse ( $337 \mathrm{~nm}$ ) focused to $200 \mu \mathrm{m}$ at a pulse energy of $\sim 30 \mu \mathrm{J}$. A large fraction of the porphyrins was neutral and could be detected using multiphoton ionization by a second pulsed uv laser beam $(\lambda=266 \mathrm{~nm}$, spot diameter $1 \mathrm{~mm}$, pulse energy $60 \mu \mathrm{J}$, pulse duration $5 \mathrm{~ns}$ ). The most probable velocity of the emerging porphyrin beam was rather slow $(270 \mathrm{~m} / \mathrm{s})$ in TLD.

This direct ablation scheme is also successfully applied to tripeptides and other small biomolecules but it is generally believed not to work with biomolecules beyond $\mathrm{m}=2000 \mathrm{u}$, mainly because of their thermo-lability. As we will see it may however be extended to larger masses in combination with suitable cooling techniques. But let us first briefly verify if direct laser ablation of TPP already fulfills the beam criteria which we listed above.

The beam flux and detection efficiency in the present experiment were such that we could record signals as high as 3 Volt in the TOF spectrometer. Using an improved ion amplifier and integrating over a few shots it seems realistic to reach a noise level of $100 \mu \mathrm{V}$ and a signal-to-noise value of 30,000. The beam velocity of $270 \mathrm{~m} / \mathrm{s}$ fixes the full length of a Talbot-Lau interferometer to $5.5 \mathrm{~cm}$, provided that we use diffraction gratings with a period of $257 \mathrm{~nm}$, as currently available in the lab. The beam divergence for such a short interferometer with a detector width of $1 \mathrm{~mm}$ corresponds to $18 \mathrm{mrad}$. This is acceptable for an interferometer with three thin $(<500 \mathrm{~nm})$ material gratings. If we replaced the central nanofabricated grating by an optical phase grating $[15,26]$, the divergence would have to be limited to $2 \mathrm{mrad}$ in order to guarantee that the molecules do not cross the optical wells in the transverse direction. The longitudinal velocity spread should be limited to $\Delta v / v<10 \%$. With a distance of say $100 \mathrm{~mm}$ between the source and the detector the velocity selection is already $1 \%$, which is certainly good enough. The mechanical stability of such a compact system should be superior to the existing interferometer setups. The monodispersity of the beam is already rather good and it is not really required because of the mass selection in the TOF-detector. The method is also inherently vacuum compatible. The sample powder is situated in high vacuum and can be coupled to an ultra-high vacuum system. Also the neutrality condition is clearly fulfilled. Finally we have to take into account the following signal-reducing factors: The improved velocity selection will lower the present signal by a factor of three. The increased distance between source and detection enters quadratically and adds another factor of nine. The transmission of a set of three material gratings reduces the signal by a factor of roughly 125 . Replacing the central nanofabricated grating by an optical phase grating will improve the transmission by a factor of five but it requires also a ten-fold improved beam collimation.

Summarizing, it seems that an interferometer for biodyes and possibly small polypeptides (such as the tripeptide Tyrosin-Tryptophan-Glycin) could already be realized using the present source characteristics. Interferometry with polypeptides offers two interesting new perspectives to matter interferometry as they possess both a handedness and a dipole moment. Their influence on decoherence has not yet been evaluated in any experiment but it is expected to be also relevant for larger polypeptides and proteins. 


\section{Extensions of laser desorption}

Laser desorption of larger biomolecules seems to require additional cooling methods in order to suppress thermal fragmentation of these labile, floppy objects. Up to now two standard technics have been developed: Matrix assisted laser desorption (MALD) reduces the heat transfer to the analyte molecules by embedding them into an organic matrix which absorbs most of the desorbing laser energy and which entrains and cools the analyte molecules in its own thermal expansion. In contrast to that, jet expanded thermal laser desorption (JETLD) starts with desorption from a pure analyte sample which is then cooled by a stream of cold and rapidly expanding noble gas.

a. Matrix assisted laser desorption Here the analyte molecules, such as the proteins of interest, are embedded into an organic matrix for example dihydroxy benzoic acid, DHB, with a typical concentration below $c=10^{-3}$. The incident laser light is then mainly absorbed by the matrix molecules which evaporate and carry the large analyte molecules with them. The expansion process provides also sufficient cooling so that MALDI is actually capable of volatilizing singly charged thermolabile molecules with masses well beyond $10^{5}$ amu.

Our own setup is presently optimized for particles up to $50000 \mathrm{amu}$, which includes also protein clusters. This is demonstrated in Fig 6 which shows a series of bovine insulin including a range of polymers between the monomer at $5735 \mathrm{amu}$ up to the hexamer at $34400 \mathrm{amu}$.

The matrix not only cools but also enhances the ionization of the embedded molecules. Still, more than $99 \%$ of the analyte molecules remain neutral. The vast majority of the charged fraction carries only one single charge. And only those molecules are usually studied in mass spectroscopy, whereas the neutral fraction is the interesting part for molecule interferometry.

MALD thus has some very promising properties but it also poses several important challenges for quantum optical applications. First of all, the beam divergence is usually rather high[27], typically up to $1 \mathrm{rad}$. In contrast to the ions which can be collected and guided by electric fields, the neutrals therefore spread out and their flux decreases roughly quadratically with the distance from the desorption region. Transverse cooling of molecular beams is still a challenge, and collimat-

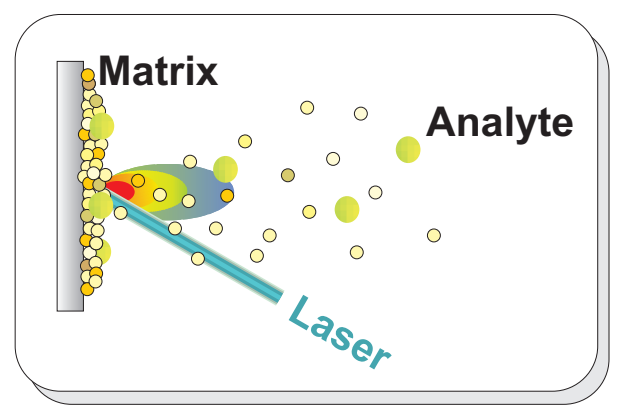

FIG. 5: Principle of matrix assisted laser desorption. ing the beam to below $1 \mathrm{mrad}$ is therefore tantamount to cutting the signal by a factor of $10^{6}$. Also, the typical beam velocity and velocity spread is rather high, with $v=200 \ldots 1000 \mathrm{~m} / \mathrm{s}$ and $\Delta v \geq 300 \mathrm{~m} / \mathrm{s}$. Selecting to $\Delta v / v \sim 1 \%$ will therefore further reduce the signal by a factor 100 . The presence of three small-slit interference gratings would enter again with the factor 125 as before. With a surface density of $10^{9} / \mathrm{cm}^{2}$ analyte molecules in a source monolayer, 5 desorbed monolayers per shot and 10 shots per second and a desorption area of about $1 \mathrm{~mm}^{2}$, we expect of the order of 0.01 molecules per second in the detector. Even if the detector can fully exploit the beam angle and if it has a detection efficiency close to unity, the integration time would be too long to keep the interferometer stable. However, several parameters still have to and can be improved, among them the particle density in the source, the repetition rate of the desorption process and probably also the transverse temperature.

b. Jet expanded laser desorption, JETLD, The analyte density can be easily enhanced by three to four orders of magnitude if we omit the matrix. And the molecules can still be cooled if we add a supersonic expansion of a carrier which entrains the desorbed proteins. JETLD is successfully used by various groups (e.g. [28, 29]) up to particles as large as insulin [30]. The main advantage of this method is the overall collisional cooling. The internal temperature can be significantly lowered, the beam divergence is reduced and also the longitudinal velocity spread can shrink down to a few percent. However, the external carrier gas cannot reduce the longitudinal velocity which even for cold and heavy noble gases is limited to above $300 \mathrm{~m} / \mathrm{s}$. But recent developments of a new variant of Talbot Lau interferometry [15] with mixed material and optical gratings ('MOM-TLI') with periods in the range of $250 \mathrm{~nm}$ are rather promising and are expected to enable nearfield interference with objects in the mass range of insulin, up to velocities as high as $300 \mathrm{~m} / \mathrm{s}$.

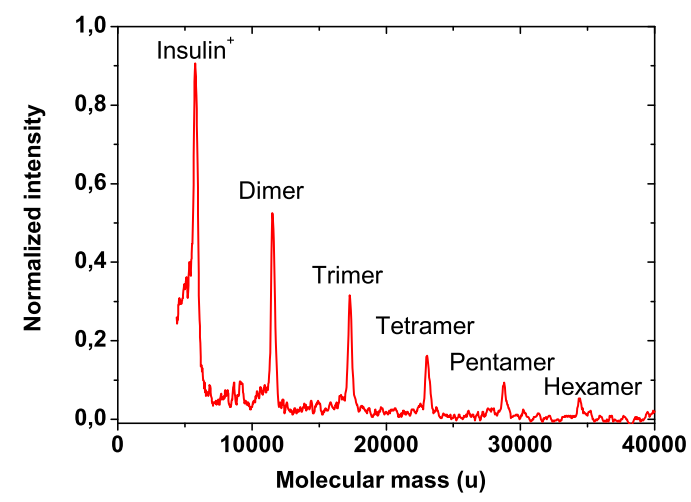

FIG. 6: MALDI of insulin: The full insulin series from the monomer up to the hexamer can be observed as quite monodisperse singly charged ions. 


\section{Electrosprays}

Electrospray Ionization (ESI) is the second of the two main standard techniques for generating macromolecular beams [25, 31]. Due to its non-thermal, soft character it allows volatilizing proteins, nanocrystals and even whole viruses [32] without any major fragmentation.

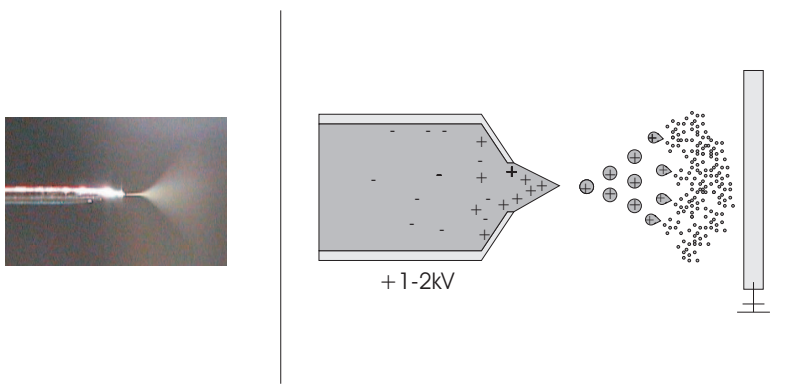

FIG. 7: Electrospray process. Highly charged droplets are formed at the tip of the electrospray needle. Repeated evaporation of the solvent and Coulomb fissions rapidly reduce the size of the droplets.

For this method, analyte molecules are dissolved in a polar liquid at concentrations of typically $\mathrm{c}=1 \times 10^{-6} \ldots 1 \times 10^{-3}$ and filled into a thin needle with an inner tip diameter of a few $\mu \mathrm{m}$. The needle is connected to a voltage of $1 \ldots 4 \mathrm{kV}$ and positioned in a few millimeters distance to the vacuum inlet hole which also serves as a counter electrode. At high electric fields an imbalance between electro- and hydrodynamical forces finally leads to the formation of a spray. Figure 7 shows both a photograph and a sketch of this process. The emerging droplets typically have diameters below $1 \mu \mathrm{m}$ and they are highly charged. A repeated cycle of 'solvent evaporation' and 'charge induced fission' finally leads to the preparation of many isolated, highly charged and airborne molecules [31]. For many biomolecules the mass to charge ratio is situated in the range of $m / z=1000 \ldots 2000 \mathrm{u} / \mathrm{e}$. Correspondingly we detect insulin $(\mathrm{m}=5735 \mathrm{u})$ with between two and six charges on a single molecules. For the green fluorescent protein $(\mathrm{GFP}, \mathrm{m}=27000 \mathrm{u}$ ) we even observe up to 24 charges on a single particle as shown in Fig. 8. Electrosprays can be operated in either a positive or negative mode, and desolvate either protonated (positive) or deprotonated (negative) biomolecules [33].

Electrospray is a source which explicitly requires ambient conditions. The heat transfer between the surrounding air and the solvent droplets is needed to speed up their evaporation. The detection and characterization of the charged particles is done using quadrupole mass spectroscopy (QMS). As the spectrometer is operated under high vacuum the molecular ions have to be transferred through a three-stage differential pumping system, as indicated in Fig. 9. An Einzellens system guides and focuses the ions to the entrance lens of the QMS.

The successful recording of the spectra in Fig. 8 shows that this can be done rather efficiently - with more than ten million insulin ions per second detected by the mass spectrometer. Compared to the initial flux of molecules of $4.0 \times 10^{10}$ particles $/ \mathrm{s}$ - calculated from a concentration of $\mathrm{c}=1 \times 10^{-5} \mathrm{~mol} / \mathrm{l}$

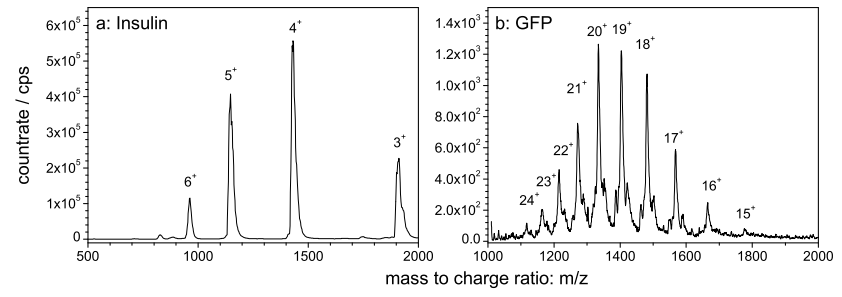

FIG. 8: ESI-QMS Spectra of a: Insulin and b: green fluorescent protein (GFP). Both molecules show a mass-to-charge distribution ranging between $1000 \ldots 2000 \mathrm{u} / \mathrm{e}$.

and a given flow rate of $0.4 \mu \mathrm{l} / \mathrm{min}$ - we detect up to $10^{-3}$ of all sprayed particles in the mass spectrometer. This is a conservative number, as the detection efficiency in particular of massive ions (e.g. GFP) is clearly below unity. More sophisticated ion optics in the various pumping stages promises not only to improve the total ion flux but also the transverse velocity spread by an estimated order of magnitude [34, 35]. However, ultimate cooling of the molecular ions will require more advanced techniques such as for instance a cryogenically cooled multi-pole ion trap.

We see that some important source criteria can be fulfilled by electrosprays. We can couple a large number of very massive particles, mass selected into high vacuum. The prospects for slowing and cooling are even more promising for ions than for neutrals. However, the main challenge in ESI based experiments on quantum optics is to generate neutral particles. Ion interferometry is not rendered impossible by any fundamental law of nature. And indeed, electron [36, 37] and $\mathrm{He}^{+}$. interferometry [38] have been successfully demonstrated. But neutralization is currently believed to be essential as the small diffraction angles of very massive and slow objects might be easily hidden behind inhomogeneous beam deflections caused by even very tiny electric stray fields.

\section{Neutralization concepts for Electrospray}

The emergence of neutral molecules out of the spray process is still a matter of debate. Although it is obvious, that

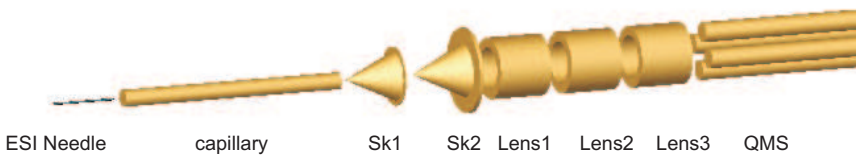

FIG. 9: Electrospray Vacuum Interface: Three differentially pumped vacuum stages couple the electrospray at atmospheric pressure to a quadrupole mass spectrometer in high vacuum. The electrosprayed ions are guided by an Einzellens system. 
not all dissolved molecules contribute to the measured spray current, it appears that the majority of neutral particles must be hidden in remaining liquid droplets or as larger clusters. Indication for both can be observed when we spray fluorescent molecules. And up to now we were not able to detect any unambiguous proof for a significant remaining fraction of isolated neutral molecules in the beam. But it may even turn out to be advantageous to work with charged particles in the preparation phase and to neutralize them subsequently in air and/or in vacuo.

Very efficient charge reduction methods have already been developed in aerosol physics for macromolecules up to the size of viruses [32, 39]. Charge reduction is achieved via proton transfer collisions between the electrosprayed particles and a bipolar ion cloud at atmospheric pressure. This is a very soft technique, which does not lead to any significant fragmentation [40] and which allows to choose the mean charge state per molecule in a rather controlled way.

However, as mentioned above, a complete neutralization in air is not desired as this removes the most efficient handle for guiding, cooling and slowing of the molecules in vacuum. Also the coupling efficiency to the high vacuum chamber is dramatically reduced and the molecular total velocity is significantly increased if one utilizes airborne neutrals compared to airborne ions.

It therefore appears advantageous to implement two steps, using bipolar air for reducing the average charge per molecule from e.g. $24^{+}$to $1^{+}$at atmospheric pressure, and an in vacuo mechanism to remove or add a last proton or electron. The first of the two steps has recently been implemented in our lab and is currently being optimized.

Various neutralization principles are conceivable in vacuo, among them ion-ion charge exchange, ion-neutral charge exchange, electron attachment, electron detachment (using either laser radiation or electron impact), as well as molecule/surface charge exchange.

All these schemes still have to be compared with respect to four criteria: Firstly, the neutralization should be highly efficient. Secondly, the structure of the cluster/molecule must not be modified e.g. by neutralization induced fragmentation. Thirdly, the momentum transfer during the charge reduction must be minimal in order to allow the emission of a welldirected neutral molecular beam e.g. out of a pre-cooled ion trap. Finally, one has to fulfill the previously discussed vacuum compatibility requirements.

Although some of the mentioned neutralization schemes have already been described in the literature many experimental studies are still needed to identify the most appropriate technique for future applications in quantum optics.

\section{CONCLUSIONS}

We have demonstrated the wave nature of large complex molecules, such as fullerenes and biodyes using near-field Talbot Lau interferometry. We have identified important decoherence mechanisms for matter waves but we also learn from our quantitative experiments that neither collisions nor thermal radiation should limit our experiments - at least up to masses in the range of one million mass units. Our discussion of the technological issues related to novel sources for quantum optics shows that there is hope to apply laser desorption to medium sized particles, possibly up to small proteins, such as insulin. For large proteins, clusters or nanocrystals ion traps might offer a way to cool these particles, certainly to cryogenic temperatures (i.e. $4 \mathrm{~K}$ ), and potentially much lower using cavity assisted laser cooling schemes [41]. But much interesting work lies still ahead on the way towards quantum interference of massive particles.

\section{ACKNOWLEDGMENTS}

This work is supported by the Austrian FWF under START Y177, SFB F1505, by the Wiener Hochschuljubilumsstiftung der Stadt Wien and by the Commission of European Communities under the contract no. HPRN-CT-2002-00309. We acknowledge the important contribution of Klaus Hornberger in the theoretical modeling of our experiments and Anton Zeilinger for his continuous support.
[1] C. Davisson and L. Germer, Nature 119, 558 (1927).

[2] I. Estermann and O. Stern, Z. Phys. 61, 95 (1930).

[3] E. Cornell and C. Wieman, Rev. Mod. Phys. 74, 875 (2002).

[4] Y. Ji, Y. Chung, D. Sprinzak, M. Heiblum, D. Mahalu, and H. Shtrikman, Nature 422, 415 (2003).

[5] C. H. V. der Wal, A. C. J. T. Haar, F. K. Wilhelm, R. N. Schouten, C. J. P. M. Harmans, T. P. Orlando, S. Lloyd, and J. E. Mooij, Science 290, 773 (2000).

[6] T. Young, Lectures on Natural Philosophy, vol. 1 (London, 1807).

[7] M. Arndt, O. Nairz, J. Voss-Andreae, C. Keller, G. V. der Zouw, and A. Zeilinger, Nature 401, 680 (1999).

[8] L. W. Bruch, W. Schöllkopf, and J. P. Toennies, Journal Of Chemical Physics 117, 1544 (2002).

[9] H. F. Talbot, Philos. Mag. 9, 401 (1836).
[10] E. Lau, Ann. Phys. 6, 417 (1948).

[11] J. F. Clauser and S. Li, Phys. Rev. A 49, R2213 (1994).

[12] J. Clauser, in Experimental Metaphysics, edited by R. Cohen, M. Horne, and J. Stachel (Kluwer Academic, 1997).

[13] L. Hackermüller, S. Uttenthaler, K. Hornberger, E. Reiger, B. Brezger, A. Zeilinger, and M. Arndt, Phys. Rev. Lett. 91, 90408 (2003).

[14] K. Patorski, in Progress in Optics XXVII, edited by E. Wolf (Elsevier Science Publishers B. V., Amsterdam, 1989), pp. 2 108.

[15] B. Brezger, M. Arndt, and A. Zeilinger, J. Opt. B: Quantum Semiclass. Opt. 5, S82 (2003).

[16] B. Brezger, L. Hackermüller, S. Uttenthaler, J. Petschinka, M. Arndt, and A. Zeilinger, Phys. Rev. Lett. 88, 100404 (2002).

[17] K. Hornberger, S. Uttenthaler, B. Brezger, L. Hackermüller, 
M. Arndt, and A. Zeilinger, Phys. Rev. Lett. 90, 160401 (2003).

[18] K. Hornberger and J. E. Sipe, Phys. Rev. A 68, 012105 (2003).

[19] L. Hackermüller, K. Hornberger, B. Brezger, A. Zeilinger, and M. Arndt, Nature 427, 711 (2004).

[20] O. Nairz, M. Arndt, and A. Zeilinger, J. Mod. Opt. 47, 2811 (2000).

[21] L. Hackermüller, K. Hornberger, B. Brezger, A. Zeilinger, and M. Arndt, Appl. Phys. B 77, 781 (2003).

[22] G. Scoles, D. Bassi, U. Buck, and D. Lainé, eds., Atomic and Molecular Beam Methods, vol. I (Oxford University Press, 1988).

[23] E. Arimondo, W. D. Phillips, and F. Strumia, eds., Laser Manipulation of Atoms and Ions, vol. CXVIII (North-Holland, Amsterdam, 1992).

[24] M. Karas and F. Hillenkamp, Anal. Chem. 60, 2299 (1988)

[25] J. B. Fenn, M. Mann, C. K. Meng, S. F. Wong, and C. M. Whitehouse, Science 246, 64 (1989).

[26] O. Nairz, B. Brezger, M. Arndt, and A. Zeilinger, Phys. Rev. Lett. 87, 160401 (2001)

[27] A. A. Puretzky, D. B. Geohegan, G. B. Hurst, M. V. Buchanan, and B. S. Lukyanchuk, Phys. Rev. Lett. 83, 444 (1999).

[28] M. Dey, F. Moritz, G. H. Atkinson, J. Grotemeyer, and E. Schlag, J. Chem. Phys. 95, 4584 (1991).

[29] P. Dugourd, I. Compagnon, F. Lepine, R. Antoine, D. Rayane, and M. Broyer, Chemical Physics Letters 336, 511 (2001).
[30] C. Weickhardt, L. Draack, and A. Amirav, Anal. Chem. 75, 5602 (2003).

[31] R. B. Cole, Electrospray Ionization Mass Spectrometry (Wiley, New York, 1997).

[32] G. Bacher, W. W. Szymanski, S. L. Kaufman, P. Zöllner, D. Blaas, and G. Allmaier, J. Mass Spectrom. 36, 1038 (2001).

[33] R. D. Smith, J. A. Loo, C. G. Edmonds, C. J. Barinaga, and H. R. Udseth, Anal. Chem. 62, 882 (1990).

[34] A. W. Colburn, A. E. Giannakopulos, and P. J. Derrick, Eur. J. Mass Spectrom. 1, 149 (2004).

[35] S. A. Shaffer, A. Tolmachev, D. C. Prior, G. A. Anderson, H. R. Udseth, and R. D. Smith, Anal. Chem. 71, 2957 (1999).

[36] G. Moellenstedt and C. Z. Joensson, Z. Phys. 155, 472 (1959).

[37] C. Jönsson, D. Brandt, and S. Hirschi, Am. J. Phys. 42, 4 (1974).

[38] F. Hasselbach and U. Maier, Quantum Coherence and Decoherence -ISQM- Tokyo pp. 299-302 (1998).

[39] M. Scalf, M. S. Westphall, J. Krause, S. L. Kaufman, and L. M. Schmith, Science 283, 194 (1999).

[40] M. Scalf, M. Westphall, and L. M. Smith, Anal. Chem. 72, 52 (2000).

[41] P. Horak, G. Hechenblaikner, K. M. Gheri, H. Stecher, and H. Ritsch, Phys. Rev. Lett. 79, 4974 (1997). 